\title{
Eigenmode analysis of the scattering matrix for the design of MRI transmit array coils
}

\author{
Ehsan Kazemivalipour $^{1,2}$ (D) ｜ Alireza Sadeghi-Tarakameh ${ }^{1,2}$ (D) | Ergin Atalar $^{1,2}$ (D) \\ ${ }^{1}$ Department of Electrical and Electronics Engineering, Bilkent University, Ankara, Turkey \\ ${ }^{2}$ National Magnetic Resonance Research Center (UMRAM), Bilkent University, Ankara, Turkey
}

\section{Correspondence}

Ergin Atalar, Department of Electrical and Electronics Engineering, Bilkent University, 06800 Bilkent, Ankara, Turkey.

Email: ergin@ee.bilkent.edu.tr
Purpose: To obtain efficient operation modes of transmit array (TxArray) coils using a general design technique based on the eigenmode analysis of the scattering matrix. Methods: We introduce the concept of modal reflected power and excitation eigenmodes, which are calculated as the eigenvalues and eigenvectors of $\mathbf{S}^{\mathbf{H}} \mathbf{S}$, where the superscript $\mathbf{H}$ denotes the Hermitian transpose. We formulate the normalized reflected power, which is the ratio of the total reflected power to the total incident power of TxArray coils for a given excitation signal as the weighted sum of the modal reflected power. By minimizing the modal reflected power of TxArray coils, we increase the excitation space with a low total reflection. The algorithm was tested on 4 dual-row TxArray coils with 8 to 32 channels.

Results: By minimizing the modal reflected power, we designed an 8-element TxArray coil to have a low reflection for 7 out of 8 dimensions of the excitation space. Similarly, the minimization of the modal reflected power of a 16-element TxArray coil enabled us to enlarge the dimension of the excitation space by $50 \%$ compared with commonly employed design techniques. Moreover, we demonstrated that the low total reflected power for some critical excitation modes, such as the circularly polarized mode, can be achieved for all TxArray coils even with a high level of coupling.

Conclusion: Eigenmode analysis is an efficient method that intuitively provides a quantitative and compact representation of the coil's power transmission capabilities. This method also provides insight into the excitation modes with low reflection.

\section{K E Y W O R D S}

eigenmode analysis, modal reflected power, total reflected power, transmit (TxArray) array coil

\section{1 | INTRODUCTION}

RF transmit array (TxArray) coils are being extensively utilized in ultrahigh field MRI to overcome different issues, such as RF excitation inhomogeneities. ${ }^{1-8}$ TxArray coils can be very useful for conventional high-field scanners because they provide additional degrees of freedom to the designers of pulse sequences and enable RF shimming while attempting to suppress specific absorption rate ${ }^{9-11}$ hotspots. Besides, these degrees of freedom are useful for increasing the power efficiency $^{12-14}$ and accelerating RF-intense applications. ${ }^{15}$ TxArray coils can also be beneficial for the implementation of the implant-friendly mode. ${ }^{16-19}$

The performance of TxArray coils significantly profits from parallel transmit technology if the coil designs satisfy particular requirements, such as low mutual coupling between individual array elements and sufficient interaction with samples to ensure sufficiently high power efficiency. High coupling among array elements makes power delivery to the subject challenging and, therefore, is considered a 
major design issue. ${ }^{14} \mathrm{~A}$ variety of strategies have been tested to reduce mutual coupling, including geometrically overlapping the nearest-neighbor array elements ${ }^{20,21}$; inserting either capacitors or inductors between 2 nearest-neighbor array elements ${ }^{22-26}$; and adding a decoupling multiport network before TxArray coil ports. ${ }^{27,28}$

Increasing the number of transmit elements can enhance the capability of TxArray coils in terms of RF shimming and homogeneity. The problem of mutual coupling reduction becomes more complex as the number of array elements increases due to the need for more decoupling components and longer cables to decouple nonadjacent elements, which are distantly located. ${ }^{14,29,30}$ Considering the cost of high-power $\mathrm{RF}$ amplifiers, improvements in the incremental performance by further transmit elements with realistic assumptions of power budget for particular TxArray coil geometries and configurations should be determined. Theoretically, the power efficiency of a TxArray coil under single-channel excitation depends on the amount of power coupled to other transmit elements and is reflected back to the amplifier. The reflected power does not produce the $\mathrm{B}_{1}^{+}$field ${ }^{31}$ within the sample and may cause damage to the amplifiers. ${ }^{14,32}$ In this situation, proper matching, tuning, and decoupling of a TxArray coil produce higher transmitted power from the amplifier to the transmit elements and lower reflected power from all transmit elements. ${ }^{14,33}$ For a TxArray coil with coupled transmit elements under multichannel excitation, the total reflected power depends on the phases and amplitudes of the RF excitation signals, as well as the levels of matching, tuning, and decoupling. Although multiple studies focused on optimizing the excitation signals with various strict constraints, including the power consumption of TxArray coils, ${ }^{14,34-38}$ categorization of the inputs based on the transmitted and reflected power in TxArray coils warrants attention.

Here, we present the concept of excitation eigenmodes to achieve a quantitative and compact representation of the TxArray coil's transmission capabilities. For a given set of excitation signals, the total power transmitted to a TxArray coil can be analyzed using its scattering matrix (S-matrix), which can be determined as a relationship between the incident waves and reflected waves. The eigenmode analysis of $\mathbf{S}$-matrix provides insight into the excitation signals with a low level of reflected power. It offers a simple tool for quantifying, comparing, and optimizing the performance of the TxArray coils.

An ideal TxArray coil in terms of the power efficiency will have a zero total reflected power for all incident waves, which requires a zero $\mathbf{S}$-matrix. Due to the practical limitations and imperfections in matching and decoupling, $\mathbf{S}$-matrix cannot be zero. However, the $\mathbf{S}$-matrix elements can be minimized by adjusting the geometrical parameters or capacitor values of the coil using an optimization process. In this process, the optimization criterion is fundamental and affects the performance of the coil. ${ }^{39}$ The vast majority of studies concentrated on minimizing the magnitude of $\mathbf{S}$-matrix elements without considering the excitation signals..$^{30,40-42}$ However, the excitation signals have a significant role in determining the total transmitted and reflected power levels. ${ }^{39}$ For a given TxArray coil, the reflection can be high for some excitation signals but low for some others. Here, we propose that the eigenmode analysis, looking at the $\mathbf{S}$-matrix as a whole, captures more than just looking at the $\mathbf{S}$-matrix elements individually. In this new matrix analysis, in addition to the magnitude of the $\mathbf{S}$-matrix elements, their phases are also included, which were previously ignored. The insight provided by this approach can be used to minimize the total reflected power for larger sets of excitation signals by adjusting the TxArray coil design parameters. The novel design strategy utilized in this work demonstrates the possibility of obtaining very low total reflected power for specific sets of excitations, even for TxArray coils with a non-zero $\mathbf{S}$-matrix.

In this study, we provide the theory and concept of the $\mathbf{S}$ matrix eigenmode analysis, discuss the details of the TxArray coil design, and explain the optimization strategy used to adjust the capacitor values to minimize the total reflected power. We provide some simulation and experiment results on 4 dual-row TxArray coils with a different number of channels for imaging at 3 Tesla scanners. These coils ${ }^{43,44}$ have a significant potential to replace the conventional body coils because they can perform similar to the traditional birdcage coils ${ }^{45-47}$ if they are driven in the circularly polarized (CP) form but also provide additional degrees of freedom for many valuable applications of the TxArray coils.

\section{2 | THEORY}

The S-matrix describing a TxArray coil characterizes all the power interactions between its input ports. For a generic TxArray coil consisting of $N$ transmit elements, the $\mathbf{S}$-matrix is represented as $\mathbf{b}=\mathbf{S a}$, where $\mathbf{a}$ and $\mathbf{b}$ are the vectors of the incident and reflected waves, respectively, defined as

$$
\mathbf{a}=\frac{1}{2 \sqrt{\left|\mathfrak{R}\left\{Z_{0}\right\}\right|}}\left(\mathbf{V}+Z_{0} \mathbf{I}\right) \quad \text { and } \quad \mathbf{b}=\frac{1}{2 \sqrt{\left|\mathfrak{R}\left\{Z_{0}\right\}\right|}}\left(\mathbf{V}-Z_{0}^{*} \mathbf{I}\right) \text {, }
$$

where $\mathbf{V}$ and $\mathbf{I}$ are the vectors of RMS port voltages and currents. $Z_{0}$ is the reference impedance and is chosen as $50 \mathrm{ohms} \mathrm{in}$ this study. With this formulation, the total average power transmitted to a TxArray coil with lossless transmission lines can be written as ${ }^{48}$

$$
P_{t}^{T}=\mathbf{a}^{\mathbf{H}} \mathbf{a}-\mathbf{b}^{\mathbf{H}} \mathbf{b}=\mathbf{a}^{\mathbf{H}}\left(\mathbf{U}-\mathbf{S}^{\mathbf{H}} \mathbf{S}\right) \mathbf{a},
$$

where the superscript $\mathbf{H}$ denotes the Hermitian (conjugate) transpose, and $\mathbf{U}$ denotes the identity matrix. Equation (2) 
expresses the net transmitted power as the difference between the incident power and the reflected power, which can be quantified as $P_{i}^{T}=\mathbf{a}^{\mathbf{H}} \mathbf{a}$ and $P_{r}^{T}=\mathbf{b}^{\mathbf{H}} \mathbf{b}$, respectively. A perfect design has $100 \%$ transmitted power and no reflected power, which does not occur when the transmit coil elements are coupled. We define the normalized reflected power as the ratio of the total reflected power to the total incident power that can be characterized as a function of the excitations and $\mathbf{S}$-matrix

$$
\lambda(\mathbf{a})=\frac{P_{r}^{T}}{P_{i}^{T}}=\frac{\mathbf{b}^{\mathbf{H}} \mathbf{b}}{\mathbf{a}^{\mathbf{H}} \mathbf{a}}=\frac{\mathbf{a}^{\mathbf{H}} \mathbf{S}^{\mathbf{H}} \mathbf{S a}}{\mathbf{a}^{\mathbf{H}} \mathbf{a}} .
$$

$\lambda(\mathbf{a})$ can be minimized to ensure high transmit efficiency.

Examining $\mathbf{S}^{\mathbf{H}} \mathbf{S}$ can provide information on the total incident and reflected power of a TxArray coil. ${ }^{49,50} \mathbf{S}^{\mathbf{H}} \mathbf{S}$ is a Hermitian matrix, which is therefore diagonalizable by a unitary similarity transformation. ${ }^{51}$ Hence,

$$
\mathbf{S}^{\mathbf{H}} \mathbf{S}=\mathbf{Q} \Lambda \mathbf{Q}^{\mathbf{H}},
$$

where $\mathbf{Q}$ is a unitary matrix (ie, $\mathbf{Q} \mathbf{Q}^{\mathbf{H}}=\mathbf{Q}^{\mathbf{H}} \mathbf{Q}=\mathbf{U}$ ) formed by the eigenvectors $\left(\mathbf{q}_{n}\right)$ of $\mathbf{S}^{\mathbf{H}} \mathbf{S}$. $\Lambda$ is a diagonal matrix formed by the eigenvalues $\left(\lambda_{n}\right)$ of $\mathbf{S}^{\mathbf{H}} \mathbf{S}$.

If the TxArray coil is excited in such a way that the vector of the incident waves is equal to the $n$th eigenvector of $\mathbf{S}^{\mathbf{H}} \mathbf{S}$, that is, $\mathbf{a}=\mathbf{q}_{n}$, then the normalized reflected power yields

$$
\lambda\left(\mathbf{a}=\mathbf{q}_{n}\right)=\frac{\mathbf{b}^{\mathbf{H}} \mathbf{b}}{\mathbf{a}^{\mathbf{H}} \mathbf{a}}=\frac{\mathbf{q}_{n}^{\mathbf{H}} \mathbf{S}^{\mathbf{H}} \mathbf{S} \mathbf{q}_{n}}{\mathbf{q}_{n}^{\mathbf{H}} \mathbf{q}_{n}}=\frac{\mathbf{q}_{n}^{\mathbf{H}} \lambda_{n} \mathbf{q}_{n}}{\mathbf{q}_{n}^{\mathbf{H}} \mathbf{q}_{n}}=\lambda_{n}
$$

because $\left[\mathbf{S}^{\mathbf{H}} \mathbf{S}\right] \mathbf{q}_{n}=\lambda_{n} \mathbf{q}_{n}$. It should be noted that all eigenvalues are positive real with values less than or equal to one. ${ }^{52}$ The eigenvectors of $\mathbf{S}^{\mathrm{H}} \mathbf{S}$ are orthogonal ${ }^{52}$ and represent the excitation vectors of the TxArray coil. Therefore, they shall be called the excitation eigenmodes of the TxArray coil, and the eigenvalues of $\mathbf{S}^{\mathbf{H}} \mathbf{S}$ shall be called the modal reflected power values of the TxArray coil. The normalized reflected power in the case of an arbitrary vector of incident waves, which can be uniquely expanded as a sum of the distinct eigenmodes, that is, $\mathbf{a}=\sum_{n=1}^{N} w_{n} \mathbf{q}_{n}=\mathbf{Q w}$, where $\mathbf{w}=\left[w_{1} w_{2} \ldots w_{N}\right]^{\mathbf{T}}$ is the vector of the expansion coefficients, can be expressed as follows

$\lambda(\mathbf{a})=\frac{\mathbf{a}^{\mathrm{H}} \mathbf{S}^{\mathrm{H}} \mathbf{S a}}{\mathbf{a}^{\mathrm{H}} \mathbf{a}}=\frac{\mathbf{w}^{\mathrm{H}} \mathbf{Q}^{\mathrm{H}} \mathbf{S}^{\mathrm{H}} \mathbf{S} \mathbf{Q w}}{\mathbf{w}^{\mathrm{H}} \mathbf{Q}^{\mathrm{H}} \mathbf{Q} \mathbf{w}}=\frac{\mathbf{w}^{\mathrm{H}} \mathbf{Q}^{\mathrm{H}} \mathbf{Q} \Lambda \mathbf{Q}^{\mathrm{H}} \mathbf{Q} \mathbf{w}}{\mathbf{w}^{\mathrm{H}} \mathbf{Q}^{\mathrm{H}} \mathbf{Q} \mathbf{w}}=\frac{\mathbf{w}^{\mathbf{H}} \Lambda \mathbf{w}}{\mathbf{w}^{\mathbf{H}} \mathbf{w}}=\sum_{n=1}^{N}\left(\lambda_{n}\left(\frac{w_{n}}{\|\mathbf{w}\|}\right)^{2}\right)$.

Equation (6) shows that the normalized reflected power can be represented as the normalized weighted sum of the modal reflected power values. Because the form of $\lambda(\mathbf{a})$ is a Rayleigh quotient, ${ }^{53}$ its values are always between the smallest and largest $\lambda_{n}$ values. ${ }^{49}$ To obtains an ideal TxArray coil with zero total reflected power for any arbitrary inputs, all eigenvalues of $\mathbf{S}^{\mathbf{H}} \mathbf{S}$ must be zero, which does not appear to be feasible. However, the parameters in determining $\mathbf{S}^{\mathbf{H}} \mathbf{S}$ can be adjusted to minimize all eigenvalues and achieve a low total reflected power for a broader set of incident waves.

\section{3 | METHODS}

\section{$3.1 \mid \lambda$-opt design strategy}

In this study, we employed a design strategy based on the minimization of the normalized reflected power to obtain the capacitor values of TxArray coils, known as $\lambda$-optimization $(\lambda$-opt). This strategy minimizes the normalized reflected power for excitation eigenmodes as well as for the $\mathrm{CP}$ excitation vector, that is, $\lambda_{C P}$. Minimizing $\lambda_{C P}$ provides the opportunity to compose the CP mode from the most efficient excitation eigenmodes. However, the optimization problem was formulated as

$$
\lambda \text { - opt: } \min _{\overline{\mathbf{c}}} \sum_{n=1}^{N}\left(\alpha_{n} \lambda_{n}^{2}\right)+\alpha_{C P} \lambda_{C P}^{2},
$$

where $\overline{\mathbf{c}}$ is the vector of the capacitor values and $N$ is the total number of channels. $\alpha_{n}$ and $\alpha_{C P}$ represent the weights for each term. A constraint can be added to Equation (7) to obtain desired modes of operations. We used this general formulation under 3 specific cases:

$$
\begin{aligned}
\text { case1 } \min _{\overline{\mathbf{c}}} \frac{1}{N} \sum_{n=1}^{N} \lambda_{n}^{2}+\lambda_{C P}^{2} \\
\lambda \text { - opt: case2 } \min _{\overline{\mathbf{c}}} \frac{1}{N} \sum_{n=1}^{N} \lambda_{n}^{2}+\lambda_{C P}^{2} \\
\text { s.t. } \quad \lambda_{C P} \leq 0.01 \\
\text { case3 } \quad \min _{\overline{\mathbf{c}}} \frac{1}{N} \sum_{n=1}^{N} \lambda_{n}^{2}
\end{aligned}
$$

These 3 different minimization cases were employed to investigate the impact of the added constraint and weighting coefficients on the optimization results. To compare the performance of the $\lambda$-opt strategy, we also implemented the conventional minimization strategy, ${ }^{13,33,54,55}$ which is based on minimizing the magnitude of the $\mathbf{S}$-matrix elements individually (S-opt). For this, we minimized the difference between the actual and desired $\mathbf{S}$-matrices, as described in Ref. 55. Therefore, this optimization criterion was formulated as

$$
\begin{gathered}
\text { S-opt: } \underset{\overline{\mathbf{c}}}{\min } \frac{1}{N} \sum_{n=1}^{N} \sum_{m=1}^{N}\left|s_{m n}\right|^{2} . \\
\text { s.t. }\left|s_{n n}\right| \leq-15 \mathrm{~dB}
\end{gathered} .
$$

The added constraint puts an upper limit on the diagonal elements of S-matrix. Note that $\sum_{n=1}^{N} \sum_{m=1}^{N}\left|s_{m n}\right|^{2}$, which 
is equal to the trace of $\mathbf{S}^{\mathrm{H}} \mathbf{S}$, can also be calculated as the summation of the $\lambda_{n}$ values $^{51}$; therefore, the $\mathbf{S}$-opt approach minimizes the average of the $\lambda_{n}$ values denoted as $\lambda_{a v}$ while limiting the diagonal elements of $\mathbf{S}$-matrix to be equal to or less than $-15 \mathrm{~dB}$.

\section{2 | Proposed model for RF TxArray coil}

The proposed model and schematic of dual-row $(2 \times$-channel) head TxArray coils are shown in Figure 1. These coils have a cylindrical geometry, which is composed of $N$ transmit loops with the same dimensional sizes distributed in both the circumferential direction and the z-direction and can be decoupled (approximately) by adjusting the decoupling capacitors placed between the nearest neighbors. In each loop, 5 distinct capacitor values, that is, $\overline{\mathbf{c}}=\left[c_{d r} c_{d c} c_{t} c_{m} c_{s}\right]$, mainly control the performance of the TxArray coils as free design parameters. The chosen structure for the dualrow TxArray coil enables the coil to act like a single-row degenerate birdcage TxArray coil ${ }^{22,23,43,44,56-59}$ when the mesh currents of the adjacent elements in the axial direction cancel each other in the mid-ring segments of the coil. In the $\mathrm{CP}$ excitation mode, both rows are individually excited with identical power and linearly increasing phases, and the lower-row channels are excited with a $180^{\circ}$ phase shift relative to the upper-row channels. When the CP excitation is applied to this coil, it produces a field similar to a conventional birdcage coil with a rather uniform $\mathrm{B}_{1}^{+}$field distribution in a large FOV.

\section{3 | Numerical simulations}

Four shielded dual-row TxArray coils at 3 Tesla with 4, 8, 12, and 16 transmit channels in each row were designed and simulated (Figure 1). Additionally, 4 high-pass birdcage coils at 3 Tesla composed of $4,8,12$, and 16 rungs were simulated (Supporting Information Figure S1) to compare the $\mathrm{B}_{1}^{+}$patterns of the TxArray coils in the CP excitation mode with the traditional birdcage coils. The birdcage coils were driven in the quadrature mode.

All TxArray coil and birdcage coils had a cylindrical geometry with the same dimensions (diameter of $315 \mathrm{~mm}$, length of $270 \mathrm{~mm}$, shield diameter of $408.9 \mathrm{~mm}$, and shield length of $420 \mathrm{~mm}$ ). All rings and legs were copper strips with a width of $15 \mathrm{~mm}$. All coils shown in Figure 1 were loaded with a uniform cylindrical phantom with a diameter of 160 $\mathrm{mm}$, a length of $350 \mathrm{~mm}$, a conductivity of $0.6 \mathrm{~S} / \mathrm{m}$, and a relative permittivity of 80 .
(A)

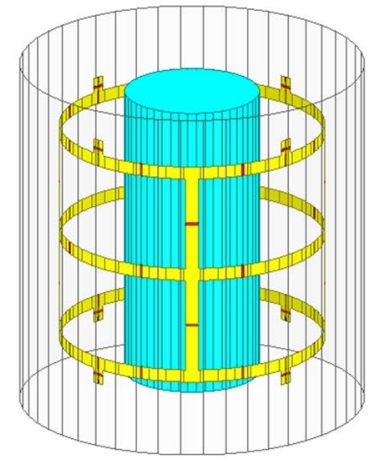

$2 \times 4-$ channel

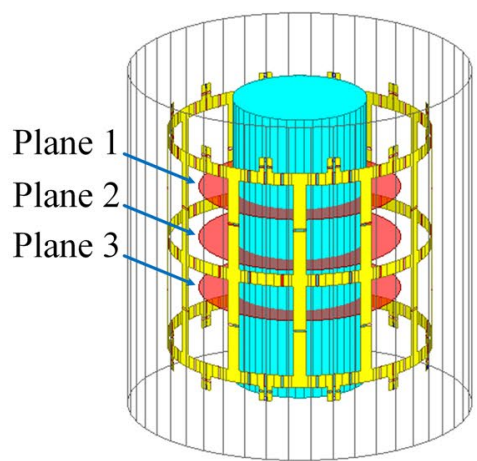

$2 \times 12-$ channel

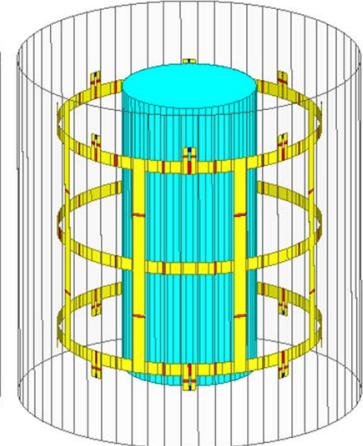

$2 \times 8$ - channel

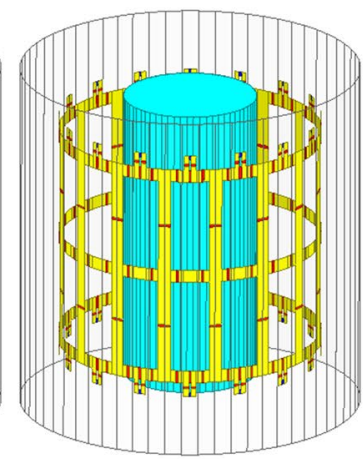

$2 \times 16$ - channel
(B)

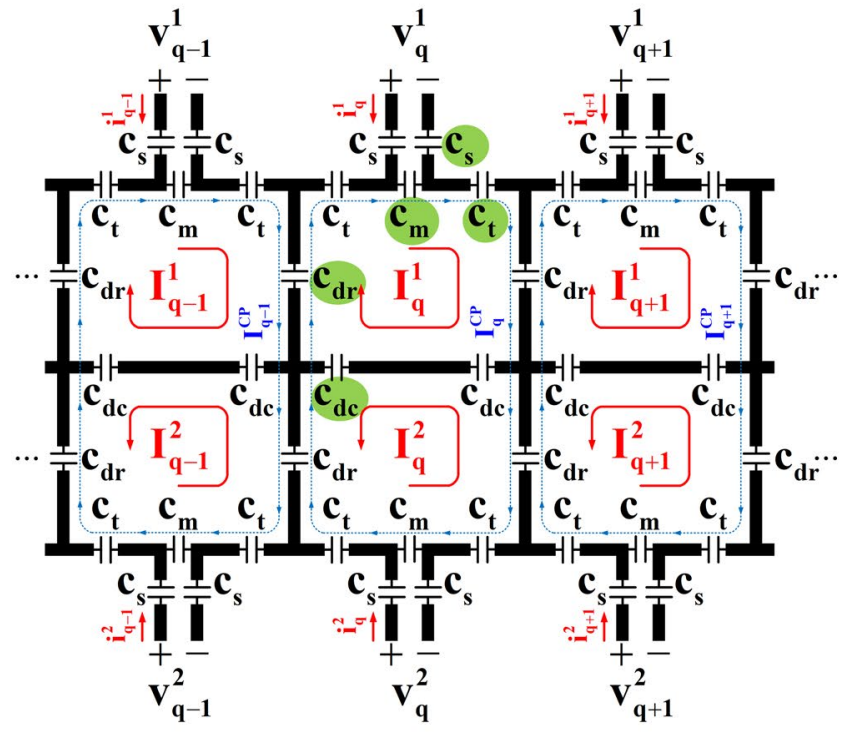

F I G URE 1 (A) EM simulation models, and (B) general schematic of dual-row degenerate birdcage TxArray coils. Three axial planes are shown in (A) to compare TxArray coil fields. Port voltages and mesh currents (red trace) are shown in each loop. $I_{q}^{C P}$ (blue trace) denotes mesh current of CP excitation mode. All coils have same dimensions, are enclosed with same cylindrical RF shields, and are loaded by same uniform cylindrical phantom. CP, circularly polarized; EM, electromagnetic; TxArray, transmit array 
Ansys Electronics Desktop 18.2 (Ansys Inc., Canonsburg, PA) was used to implement the numerical simulations at the operation frequency of $123.2 \mathrm{MHz}$. The conductors were modeled as good conductors, ${ }^{60}$ and the finite conductivity boundary was defined for them. A sphere with a radius greater than a quarter of the wavelength with the radiation boundary condition was defined as the outer surface of all simulations. Capacitors were adjusted based on the optimization method explained in the previous section using a combined finite element method and circuit analysis approach. ${ }^{61}$ By substitution of all capacitors with equivalent lumped ports, the multiport $\mathbf{S}$-matrices of the loaded TxArray coils were calculated in Ansys HFSS (Ansys Inc.). The multiport S-matrices were applied to perform the minimization problem, which was implemented in custom-written MatLab 2018b scripts (MathWorks, Natick, MA) and obtain the optimal values of the capacitors. These MatLab (Mathworks) scripts are openly available in GitHub at https://github.com/ UMRAM-Bilkent/Eigenmode-Analysis. In the next step, the multiport S-matrices were exported to ANSYS Designer (Ansys Inc.). The updated port current and voltage values were pushed back to Ansys HFSS (Ansys Inc.) to calculate the distributions of the electric and magnetic fields.

To obtain the capacitor values of the $2 \times 4$-channel TxArray coil, all 3 different minimization cases of the $\lambda$-opt method were studied. The $\lambda$-opt (case 1) approach was also used to determine the coil performance as a function of the number of channels. To evaluate the performance of the proposed optimization strategy, both the $\lambda$-opt (case 1) and $\mathbf{S}$ opt approaches were employed to optimize a $2 \times 8$-channel TxArray coil. The capacitor values in the simulation of the TxArray coils and the high-pass birdcage coils are listed in Supporting Information Table S1.
To obtain more information about the total transmitted power to each TxArray coil, the power loss in phantom and conductors, including the power loss in the shield, were calculated. The capacitors are assumed to be lossless. The radiation loss was calculated as the difference between the total transmitted power and all other losses.

The $\mathrm{B}_{1}^{+}$efficiency was evaluated as the average $\mathrm{B}_{1}^{+}$within a region of interest for a unit total incident power. For comparison of the performance of the coils, the electromagnetic fields were recorded from 3 different axial planes that pass through the phantom, as shown in Figure 1A.

\section{4 $\mid$ Measurements}

To validate the simulations, the structure of a $2 \times 4$-channel TxArray coil, which was supported by cylindrical plexiglass with a thickness of $3 \mathrm{~mm}$ and an outer diameter of $315 \mathrm{~mm}$ (Figure 2A), was constructed. The length of the coil was 270 $\mathrm{mm}$, and all rings and legs were copper strips with a width of $15 \mathrm{~mm}$. The coil's copper is broken into 56 different sections to distribute the capacitors. Due to practical limitations, the shield was built on another plexiglass in the shape of a right regular dodecagonal prism (Figure 2B). The shield is also constructed of copper strips on the outer side of the frame and slit in 12 equally spaced rectangular sections with dimensions of $105.5 \times 480 \mathrm{~mm}^{2}$ along the axial direction to reduce gradient-induced eddy currents. ${ }^{62}$ The neighboring slits were connected with three $2 \mathrm{nF}$ capacitors at positions that face rings of the coil. The strip thickness in both the coil and the shield was $35 \mu \mathrm{m}$. The coil was loaded with a cylindrical SNR phantom $\left(3.7 \mathrm{~g} / \mathrm{L} \mathrm{NiCl}_{2} \cdot 6 \mathrm{H}_{2} \mathrm{O}\right.$ and $\left.2.4 \mathrm{~g} / \mathrm{L} \mathrm{NaCl}\right)$ with a diameter of $153 \mathrm{~mm}$. MR electrical properties tomography ${ }^{63}$
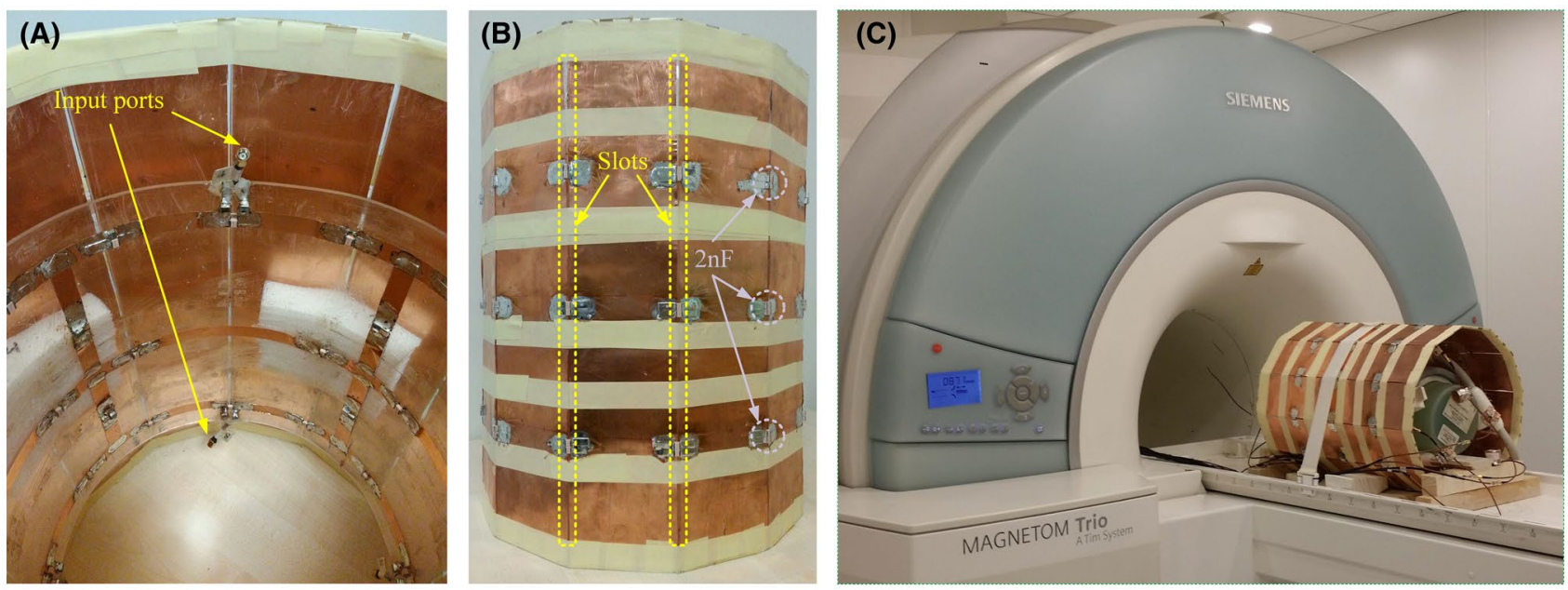

F I G U RE 2 Experimental setups. (A) $2 \times 4$-channel degenerate birdcage head TxArray coil designed and constructed using proposed method. (B) RF shield with several parallel slots placed on plexiglass in shape of right regular dodecagonal prism. (C) Overview of TxArray coil inside scanner. Coil is loaded with sodium-nickel solution phantom, and body-matrix coil of Siemens scanner (Siemens Healthcare, Erlangen, Germany) is used to pick up MR signals 
was used to measure the conductivity of the phantom as 0.62 $\mathrm{S} / \mathrm{m}$. Its relative permittivity was assumed to be equivalent to that of water. ${ }^{64,65}$

To estimate the capacitor values needed for the fabricated coil, an identical loaded $2 \times 4$-channel TxArray coil with the same $2 \mathrm{nF}$ capacitors on its shield was simulated. The capacitor values (Supporting Information Table S2) were obtained using the $\lambda$-opt (case 1) method. Note that the optimal capacitors determined by the simulation were finely tuned in the fabricated coil to minimize the error between the simulated and measured $\left|S_{n n}\right|$ values, $\lambda_{a v}$, and $\lambda_{C P}$ at $123.2 \mathrm{MHz}$.

A calibrated Agilent E5061B vector network analyzer (Agilent Technologies, Inc., Baltimore, MD, USA) was used to measure the scattering parameters of the coil. All MR experiments were conducted using a 3 Tesla scanner (Magnetom Trio, A Tim System, Siemens Healthcare, Erlangen, Germany) that was equipped with 8 transmit array channels. Each channel utilized a separate amplifier (Analogic Corp., Boston, MA) with adjustable power output with maximum peak power of 8 $\mathrm{kW}$. Accordingly, 8 coaxial cables with adjusted bazooka baluns were used to carry the RF power from the amplifiers to the TxArray coil. The TxArray coil was utilized only in transmit mode without any detuning circuits. A Siemens body-matrix coil, which is a 6-channel standard flexible surface coil with 6 integrated preamplifiers, was used as the receive coil. The possible receive performance degradation due to the lack of detuning circuits did not affect our proof of principle experiments.

A method based on the Bloch-Siegert shift ${ }^{66,67}$ was used to acquire the $\mathrm{B}_{1}^{+}$map at the central axial plane of the coil. The Bloch-Siegert shift was applied by a modified gradient-echo pulse sequence to spins by an off-resonance Fermi pulse. The duration and the off-resonance frequency were, respectively, $8 \mathrm{~ms}$ and $2 \mathrm{kHz}$. The other relevant imaging parameters were $\mathrm{TR} / \mathrm{TE}=100 \mathrm{~ms} / 12 \mathrm{~ms}$, slice thickness $=5 \mathrm{~mm}$, matrix $=$ $128 \times 128, \mathrm{FOV}=300 \mathrm{~mm}$, and number of averages $=1$. A mask with a threshold of one-tenth of the maximum $\mathrm{B}_{1}^{+}$value was applied to the $\mathrm{B}_{1}^{+}$map to minimize the effect of unreliable data (low SNR).

\section{4 | RESULTS}

\section{1 | Simulation results}

The performance of all 4 TxArray coils at $123.2 \mathrm{MHz}$ tuned by the different minimization approaches is summarized in Table 1 . In this table, all of the $\lambda_{n}$ values, $\lambda_{C P},\left|S_{n n}\right|$ values, maximum coupling levels, and $\mathrm{B}_{1}^{+}$efficiencies in the $\mathrm{CP}$ excitation mode are provided for different solutions. The simulation results based on the $\lambda$-opt (case 1) approach revealed that the ability to match and decouple the TxArray coils decreases as the number of transmit channels increases, indicated by an increase in the average of modal reflected power values, $\lambda_{a v}$. Note that this value is also equal to $\frac{1}{N} \sum_{n=1}^{N} \sum_{m=1}^{N}\left|s_{m n}\right|^{2}$. Moreover, the $\mathrm{CP}$ mode $\mathrm{B}_{1}^{+}$efficiency remains unchanged because $\lambda_{C P}$ was kept reasonably low for all 4 TxArray coils. For TxArray coils with 8, 16, 24, and 32 transmit channels, $1,7,11$, and 20 of the excitation eigenmodes have a very high modal reflected power ( $>0.93)$, respectively; therefore, these modes hardly contribute to the transmission process. $7,9,8$, and 8 of the excitation eigenmodes have a total reflection of less than $50 \%$ for coils with $8,16,24$, and 32 channels, respectively. These modes can be regarded as efficient eigenmodes of the TxArray coils, which can span the excitation space with low total reflection, and their corresponding eigenvalues are shown in bold in Table 1. Although the number of efficient eigenmodes remains relatively constant as the number of channels increases, the ratio of efficient eigenmodes to all excitation eigenmodes decreases significantly. For coils with $8,16,24$, and 32 channels, respectively, 88\%, $56 \%, 33 \%$, and $28 \%$ of the eigenmodes can be accessed efficiently $(\leq 50 \%)$, which indicates that adding channels does not necessarily lead to a proportional increase in the degrees of freedom.

The results (Table 1) also demonstrate that the solution obtained for the $2 \times 8$-channel TxArray coil by the $\mathbf{S}$-opt approach increases $\lambda_{C P}$ substantially (by 50\%) compared to the solution obtained by the $\lambda$-opt method. The increase in $\lambda_{a v}$ and decrease in $\mathrm{B}_{1}^{+}$efficiency are not very significant (13\% and 9\%, respectively). Also, 9 excitation eigenmodes with a total reflection of less than $50 \%$ are available with the solution acquired by the $\lambda$-opt (case 1) approach. In contrast, in the solution obtained by the $\mathbf{S}$-opt approach, only 6 eigenmodes have this property, indicating that the $\lambda$-opt method significantly enlarges the excitation space. For some applications that require more transmitted power or under some hardware limitations, a lower threshold value of modal reflected power can be considered for determining the excitation space. Considering an excitation space with a total reflection of less than $40 \%$ shows that 8 eigenmodes were available with the solution acquired by the $\lambda$-opt approach. In contrast, the $\mathbf{S}$-opt approach offered only 4 excitation eigenmodes.

Moreover, 3 different solutions of the $\lambda$-opt approach obtained for the $2 \times 4$-channel TxArray coil have shown that limiting $\lambda_{C P}$ to less than 0.01 (case 2) can increase $\lambda_{a v}$ significantly (by 18\%). Dropping out $\lambda_{C P}$ in the minimization problem (case 3 ) did not cause a significant change in $\lambda_{a v}$. The $2 \times 4$-channel TxArray coil has only 1 excitation eigenmode, with a reflection of more than $98 \%$ when any of the $\lambda$-opt approaches are used. Therefore, the usage of the different cases did not alter the dimension of the excitation space. Furthermore, $\lambda_{C P}$ was changed significantly with the use of different cases. The results have also shown that the $2 \times 4$-channel TxArray coil is capable of acceptable levels of matching and decoupling. 


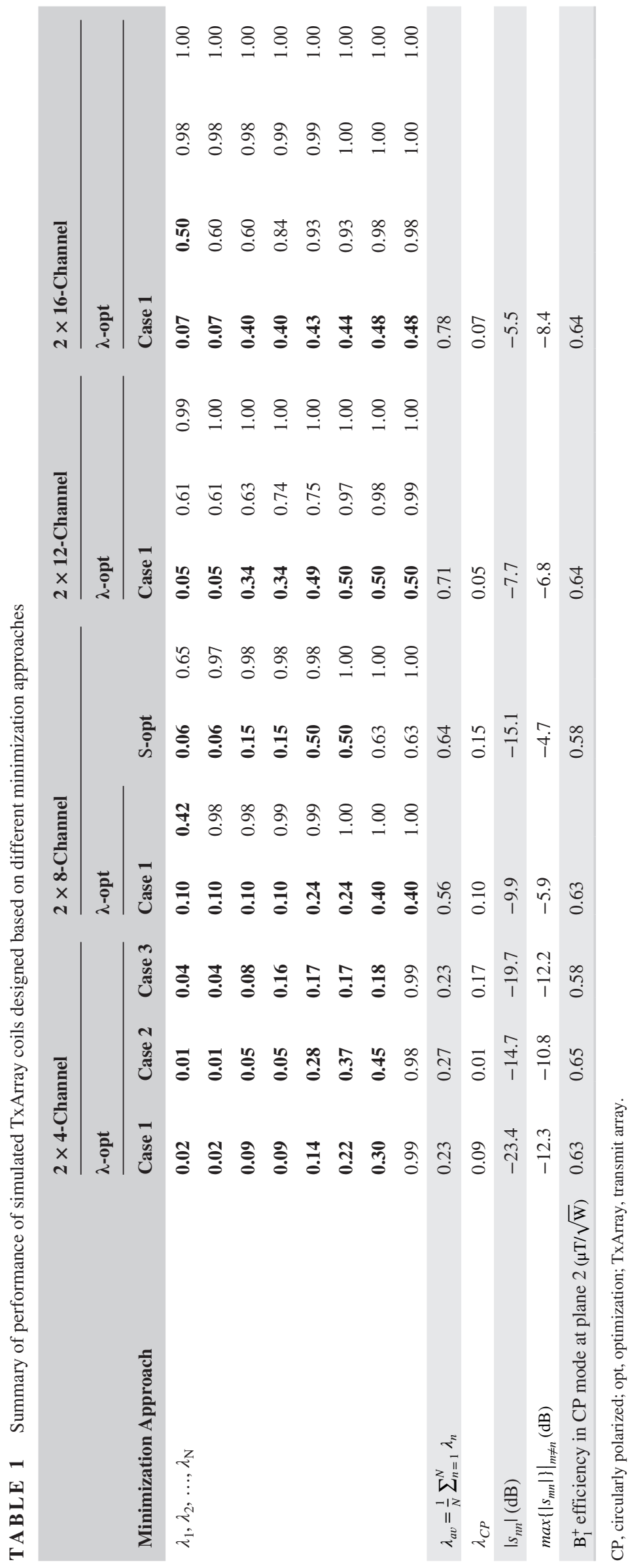


Figure 3 demonstrates the normalized reflected power, the total delivered power to the phantom, and the $\mathrm{B}_{1}^{+}$efficiency of 4 dual-row TxArray coils and 4 high-pass birdcage coils, all derived in the CP excitation mode. The TxArray coils were designed based on the $\lambda$-opt (case 1) approach. Because the number of channels increases in the TxArray coils, $\lambda_{a v}$ increases; however, $\lambda_{C P}$ remains less than $10 \%$, which is slightly worse than the high-pass birdcage coils. As can be seen in Figure 3B, more than $87 \%$ and $95 \%$ of the total incident power is delivered to the phantom for the TxArray coils and the high-pass birdcage coils, respectively, when they are used in the $\mathrm{CP}$ mode. The $\mathrm{B}_{1}^{+}$efficiencies of the TxArray coils and high-pass birdcage coils derived in the $\mathrm{CP}$ mode were also very similar (more than $0.62 \mu \mathrm{T} / \sqrt{\mathrm{W}}$ and $0.65 \mu \mathrm{T} / \sqrt{\mathrm{W}}$, respectively). Note that the slight difference between the CP performance of the TxArray coils and birdcage coils can be eradicated when the $\lambda$-opt (case 2) method is used, as can be seen in Figure 4 for the $2 \times 4$-channel TxArray coil.

The results shown in Figures 4-7 are obtained by simulating the $2 \times 4$-channel TxArray coil with the capacitors

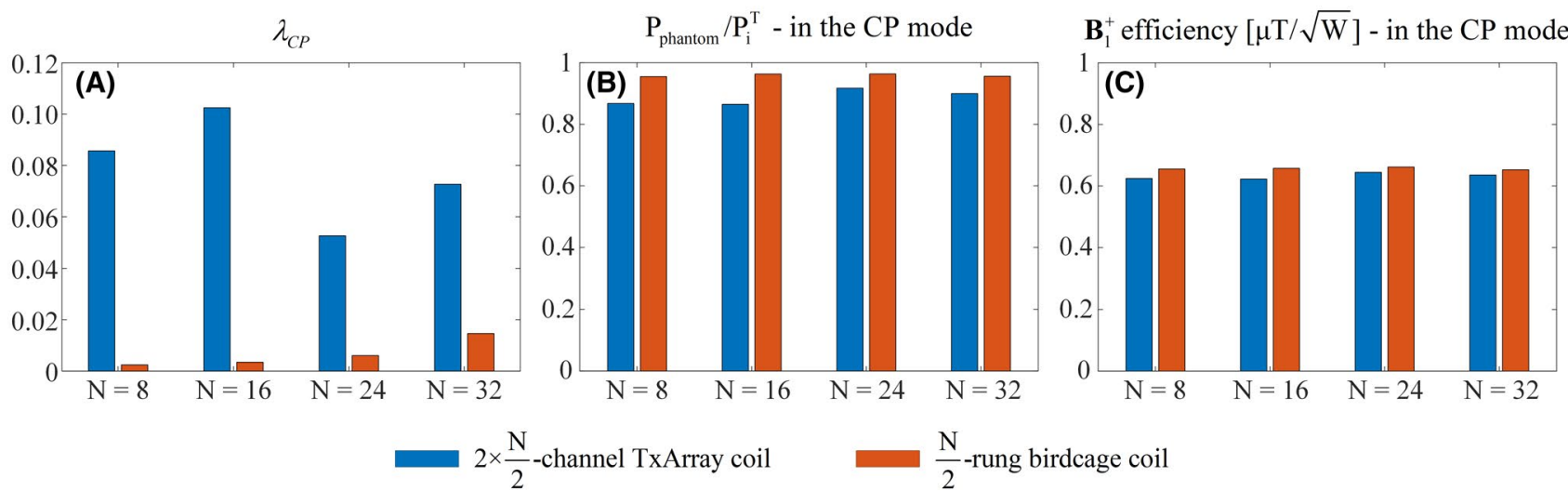

F I G URE 3 (A) Normalized reflected power; (B) total delivered power to phantom; and (C) $\mathrm{B}_{1}^{+}$efficiency of $N$-channel TxArray coil and N/2-rung high-pass birdcage coil, both derived in CP excitation mode with $N=8,16,24$, and 32. $N$-channel TxArray coils were designed based on $\lambda$-opt (case 1) approach. opt, optimization

$$
\mathbf{B}_{1}^{+} / \sqrt{\mathrm{P}_{\mathrm{i}}^{\mathrm{T}}} \text { - patterns }
$$

(A)

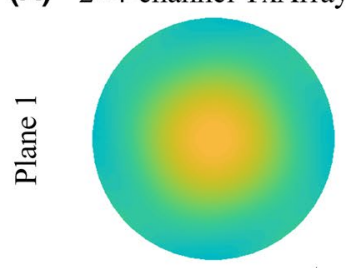

$0.582 \pm 0.006 \mu \mathrm{T} / \sqrt{ } \mathrm{W}$
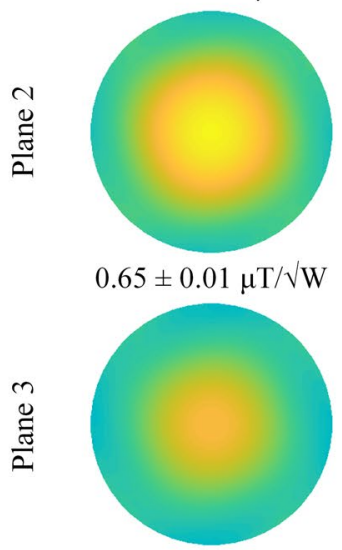

$0.582 \pm 0.006 \mu \mathrm{T} / \sqrt{ } \mathrm{W}$

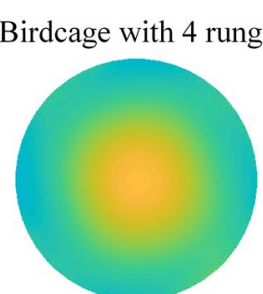

$0.587 \pm 0.006 \mu \mathrm{T} / \sqrt{W}$

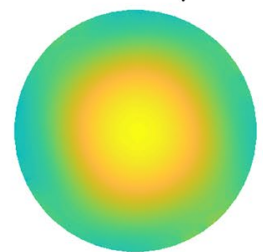

$0.655 \pm 0.01 \mu \mathrm{T} / \sqrt{ } \mathrm{W}$

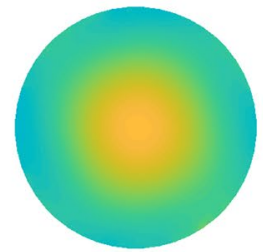

$0.587 \pm 0.006 \mu \mathrm{T} / \sqrt{ } \mathrm{W}$
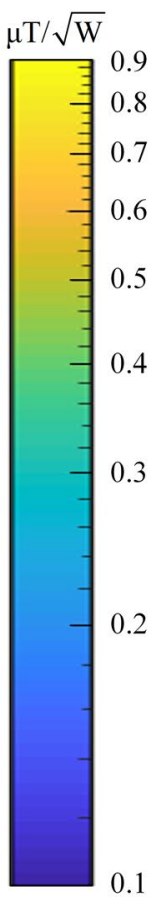

$\mathbf{E} / \sqrt{\mathrm{P}_{\mathrm{i}}^{\mathrm{T}}}$-patterns

(B) $2 \times 4$-channel TxArray Birdcage with 4 rungs $\mathrm{V} / \mathrm{m} / \sqrt{\mathrm{W}}$

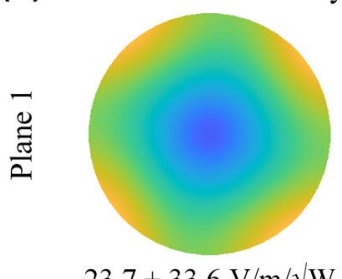

$23.7 \pm 33.6 \mathrm{~V} / \mathrm{m} / \sqrt{ } \mathrm{W}$

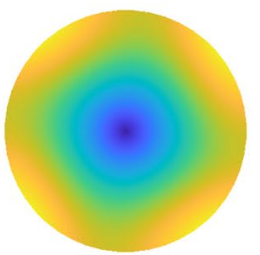

$26.3 \pm 53.8 \mathrm{~V} / \mathrm{m} / \sqrt{ } \mathrm{W}$

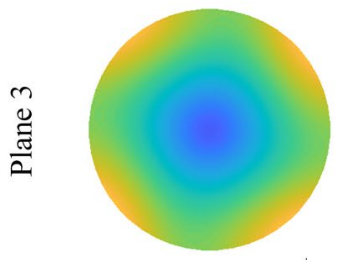

$23.7 \pm 33.6 \mathrm{~V} / \mathrm{m} / \sqrt{ } \mathrm{W}$

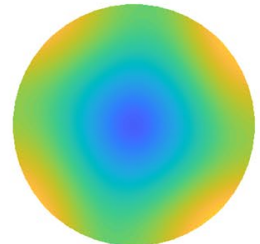

$23.9 \pm 33.5 \mathrm{~V} / \mathrm{m} / \sqrt{ } \mathrm{W}$

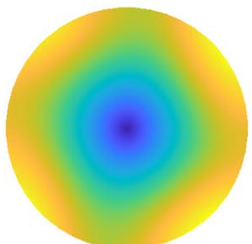

$27 \pm 54 \mathrm{~V} / \mathrm{m} / \sqrt{ } \mathrm{W}$

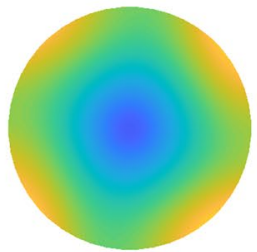

$23.9 \pm 33.5 \mathrm{~V} / \mathrm{m} / \sqrt{ } \mathrm{W}$

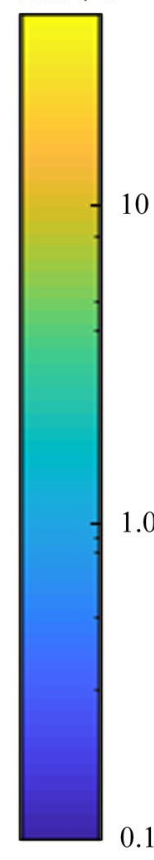

1.0

0.1

F I G URE 4 (A) $B_{1}^{+}$and (B) electric field patterns of $2 \times 4$-channel TxArray coil designed based on $\lambda$-opt (case 2) approach and 4-rung highpass birdcage coil within phantom at 3 different axial planes when both coils were driven in $\mathrm{CP}$ excitation mode. Field patterns are normalized by square root of total incident power 
determined based on the $\lambda$-opt (case 2) approach. Figure 4 also shows the $\mathrm{B}_{1}^{+}$and electric field patterns of the TxArray coil together with a 4-rung high-pass birdcage coil in the $\mathrm{CP}$

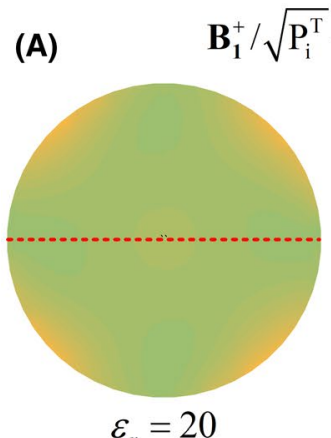

-patterns

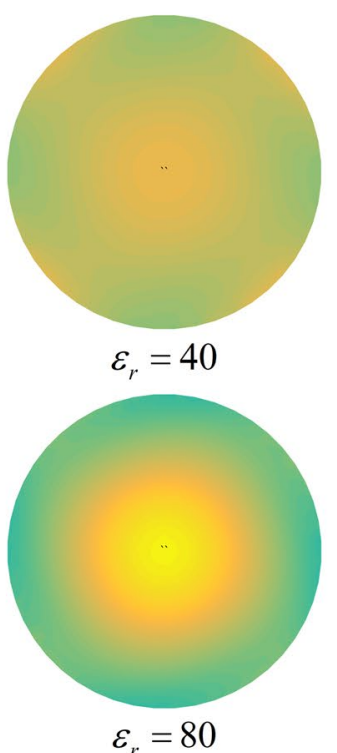

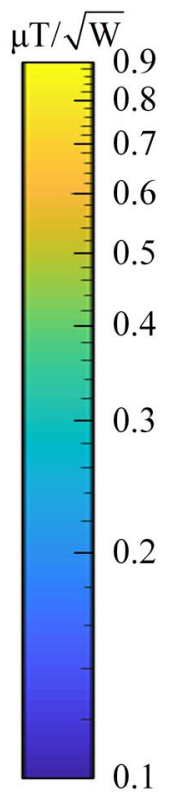

excitation mode normalized by the square root of the total incident power. Both coils generate almost identical field patterns. In Figure 5, the phantom's dielectric constant was

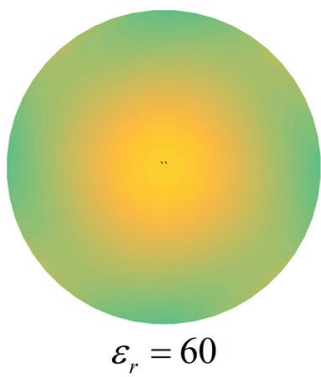

(B)

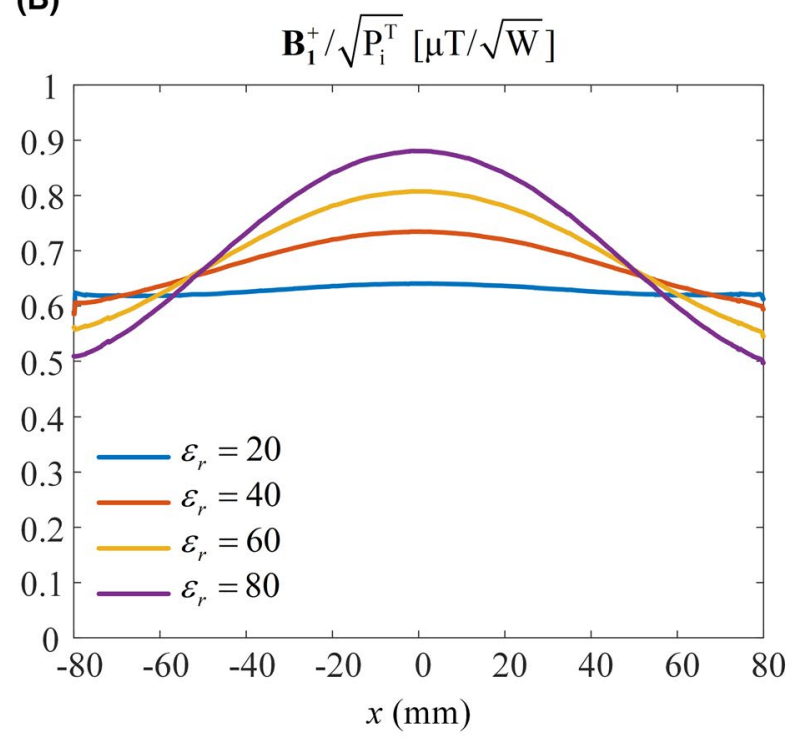

F I G U RE 5 (A) $B_{1}^{+}$patterns of $2 \times 4$-channel TxArray coil designed based on $\lambda$-opt (case 2) approach within phantom at central axial plane (plane 2) when coil was derived in $\mathrm{CP}$ excitation mode. (B) $\mathrm{B}_{1}^{+}$field distributions at $\mathrm{y}=0$. Field patterns are normalized by square root of total incident power

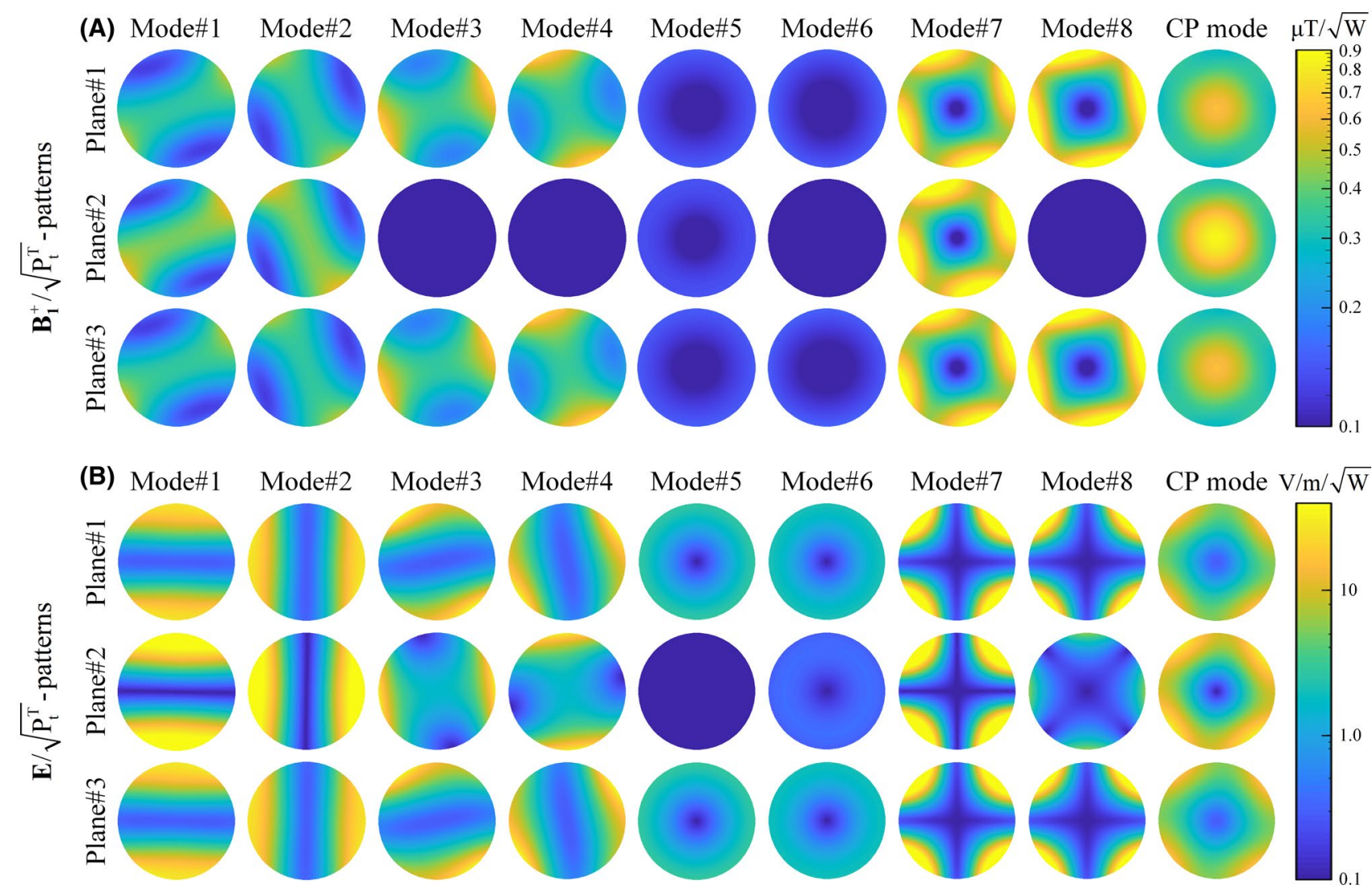

F I G U RE 6 (A) $\mathrm{B}_{1}^{+}$and (B) electric field patterns of $2 \times 4$-channel TxArray coil designed based on $\lambda$-opt (case 2 ) approach within phantom for all excitation eigenmodes; and $\mathrm{CP}$ excitation mode at planes 1,2 , and 3 . Field patterns are normalized by square root of total transmitted power 
changed between 20 and 80 to investigate the field focusing effect in the $2 \times 4$-channel TxArray coil. The coil was simulated for each dielectric constant without changing the capacitor values. Figure $5 \mathrm{~A}$ shows the $\mathrm{B}_{1}^{+}$patterns within the phantom at the central axial plane (plane 2) when the coil is derived in the CP excitation mode. Also, the field patterns reported in this figure are normalized by the square root of the total incident power. For easier comparison, Figure $5 \mathrm{~B}$ displays the normalized $\mathrm{B}_{1}^{+}$field along the $x$-axis (red dashed line shown in Figure $5 \mathrm{~A}$ ). The $\mathrm{B}_{1}^{+}$patterns for different dielectric constants demonstrate that a decrease in dielectric constant leads to a smaller field-focusing effect, and thus the $\mathrm{B}_{1}^{+}$distribution becomes more uniform. As the dielectric constant increases, significant variation in the field patterns can be observed between the center and the surrounding area. This is another indication of the birdcage coil-like behavior of the TxArray coil. The sensitivity of the $2 \times 4$-channel TxArray coil to the conductivity and diameter of the phantom is shown in Supporting Information Tables S3-S4.

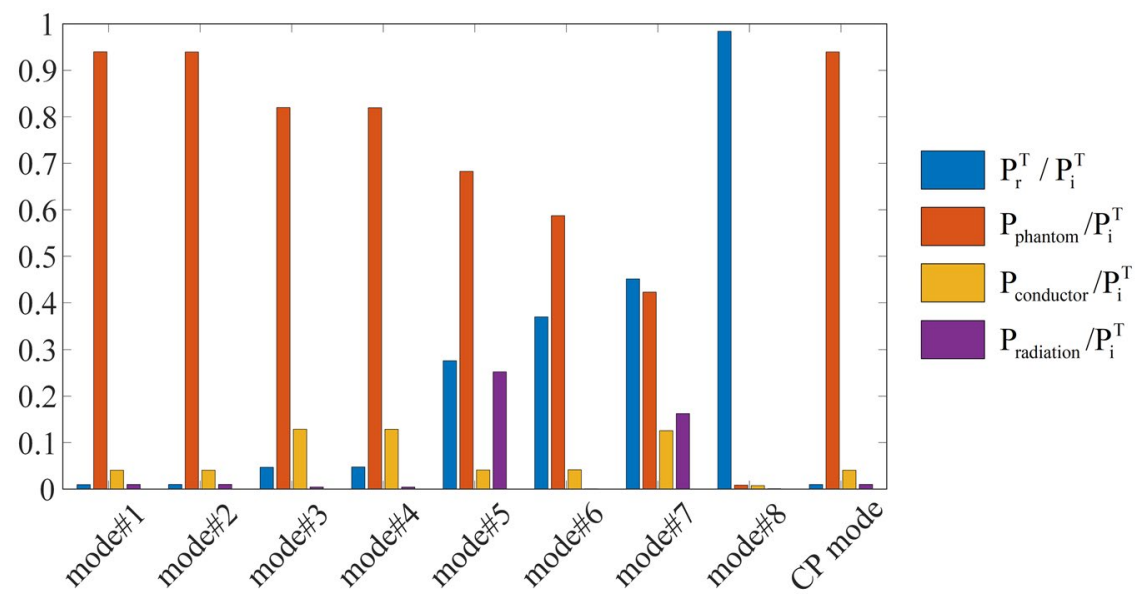

F I G URE 7 Power analysis of $2 \times$ 4-channel TxArray coil designed based on $\lambda$-opt (case 2) approach for excitation eigenmodes and $\mathrm{CP}$ excitation mode

(A) Simulated S-matrix (dB)

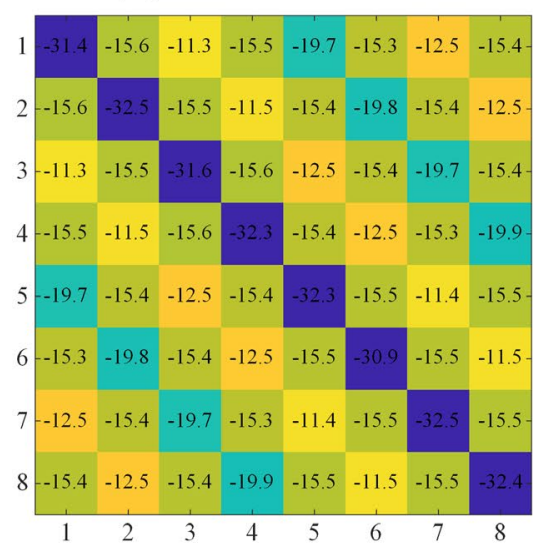

(D) $\left|s_{n n}\right|($ for $n=1,2, \ldots, 8)(\mathrm{dB})$

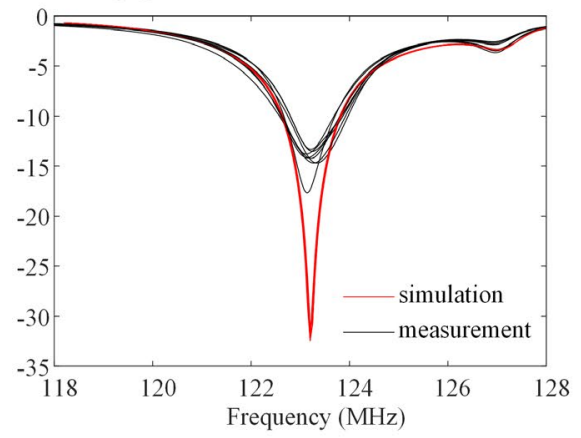

(B) Measured S-matrix (dB)

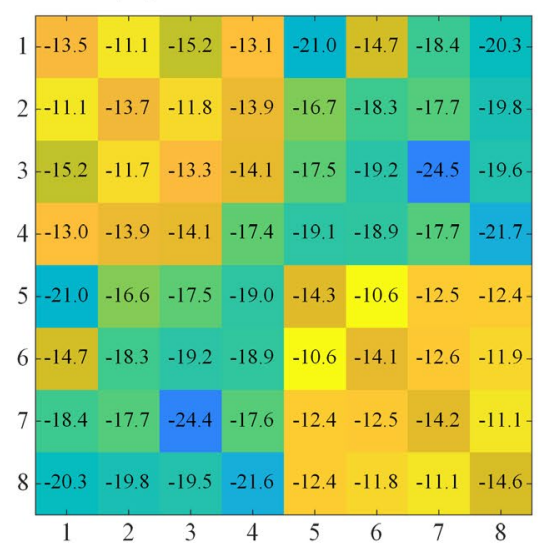

(E) Average of modal reflected power values

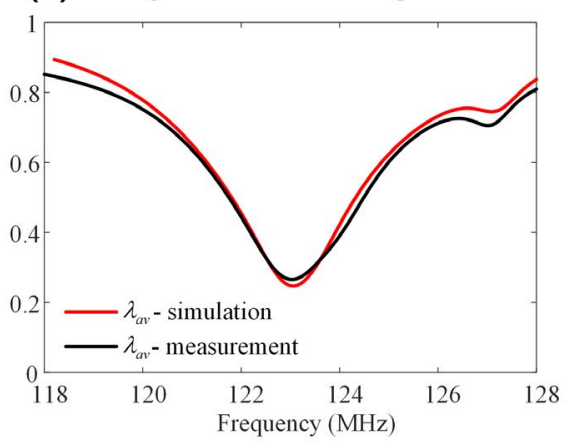

(C) Error (dB)

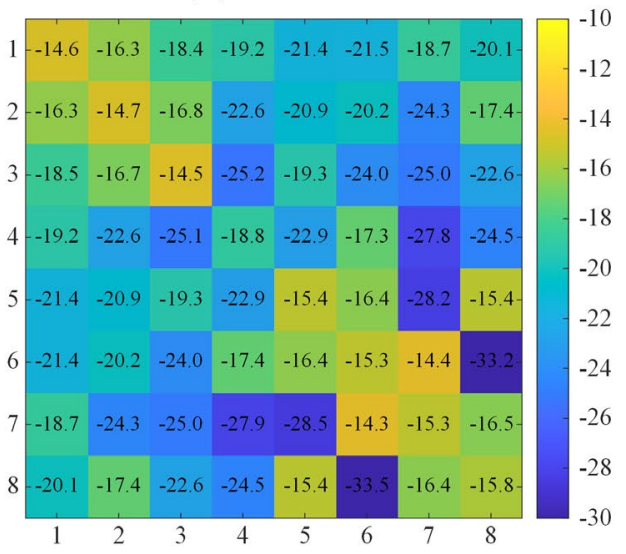

(F) The set of $\lambda_{n}$ values and $\lambda_{C P}$

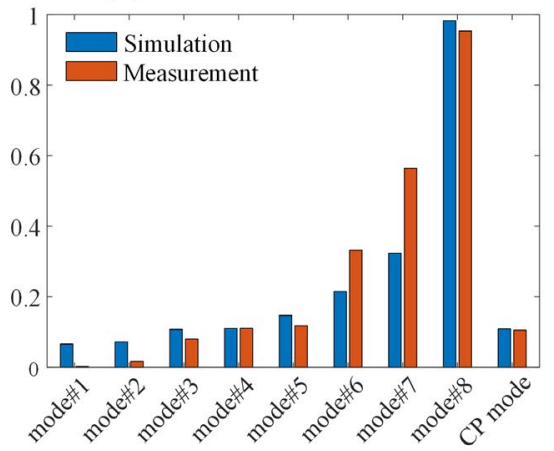

F I G URE 8 (A) Simulated and (B) measured scattering parameter matrices of loaded $2 \times 4$-channel TxArray coil designed based on $\lambda$-opt (case 1) approach at 123.2 MHz. (C) Difference between simulated and measured S-matrix. (D) $\left|S_{n n}\right|$ values for $n=1,2, \ldots, 8$ and (E) $\lambda_{a v}$ as function of frequency. (F) Modal reflected power values of TxArray coil at 123.2 MHz. For comparison, part (F) also shows $\lambda_{C P}$ 
Figure 6 shows the $\mathrm{B}_{1}^{+}$and electric field patterns of all 8 excitation eigenmodes of the $2 \times 4$-channel TxArray coil within the phantom at 3 different axial planes (Figure 1A). For comparison, the $\mathrm{B}_{1}^{+}$and $\mathbf{E}$ field patterns of the $\mathrm{CP}$ excitation mode are also shown in this figure. The field patterns are normalized by the square root of the total transmitted power. The results indicate that the first and second excitation eigenmodes produce linear fields that are perpendicular to each other. The fields generated by the $\mathrm{CP}$ excitation mode are mainly the quadrature combination of these 2 field patterns.

Figure 7 indicates where the total incident power is consumed for each eigenmode. For comparison, this figure also shows the power analysis of the $\mathrm{CP}$ mode of excitation. The total reflected power increases in higher modes for a constant total incident power, which causes less power transmission to the TxArray coil. The delivered power to the phantom is also decreased because of lower accepted power in higher modes. For the CP excitation mode, less than $1 \%$ of the incident power is reflected; $94 \%$ of the incident power is delivered to the phantom. In this mode, only $4 \%$ of the incident power is dissipated in the coil conductors, and $1 \%$ of the incident power is radiated.

\section{2 | Measurement results}

Figure 8 A,B provides the simulated and measured $\mathbf{S}$-matrix of the loaded $2 \times 4$-channel TxArray coil that was designed based on the $\lambda$-opt (case 1) approach, which demonstrates a matching level of at least $13.5 \mathrm{~dB}$ and decoupling greater than 10.5 dB. Furthermore, Figure $8 \mathrm{C}$ illustrates the error matrix that is defined as the difference between the simulated and measured S-matrix and computed as $\left|\mathbf{S}_{\text {simulated }}-\mathbf{S}_{\text {measured }}\right|$. Figure 8D,E shows the simulated and measured $\left|S_{n n}\right|$ values and $\lambda_{a v}$ as a function of the frequency. The difference in the matching and decoupling levels observed between the simulated coil and fabricated coil can be attributed to the imperfections in the construction of the coil. Figure 8F illustrates the simulated and measured modal reflected power values. For comparison, this figure also shows $\lambda_{C P}$. Figure $8 \mathrm{E}-\mathrm{F}$ reveal that the measured $\lambda_{C P}$ is in good agreement with the simulated $\lambda_{C P}$.

Figure 9 compares the $\mathrm{B}_{1}^{+}$field patterns of the fabricated and simulated $2 \times 4$-channel TxArray coil at the central axial plane (plane 2) when both coils are derived in the CP excitation mode. Phase/magnitude shimming was not performed in the measured $\mathrm{B}_{1}^{+}$pattern. As indicated in Figure 8 for the manufactured coil, the performances of all 8 transmit channels are not precisely the same; therefore, the mode generating the best $\mathrm{CP}$ excitation profile for the fabricated coil will not be the same as that defined for the simulated coil. Note that the correct $\mathrm{CP}$ excitation mode of the fabricated coil cannot be found by merely evaluating the measured scattering parameters.

\section{5 | DISCUSSION}

In this work, we defined and used the normalized reflected power as the ratio of the total reflected power to the total incident power to analyze the transmitting capabilities of TxArray coils. The excitation eigenmodes (the eigenvectors of $\mathbf{S}^{\mathrm{H}} \mathbf{S}$ ) were presented as an orthogonal basis for the TxArray coil excitation. The eigenvalues of $\mathbf{S}^{\mathbf{H}} \mathbf{S}, \lambda_{n}$, were named as the modal reflected power values. We showed that the normalized reflected power for an arbitrary excitation set, $\lambda(\mathbf{a})$, can be quantified as the weighted sum of the $\lambda_{n}$ values, which implies that the $\lambda_{n}$ values fully characterize the transmission capabilities of the TxArray coil.

In the literature for general antenna arrays, the concept of the total active reflection coefficient ${ }^{68-70}$ is used. The magnitude square of total active reflection coefficient is equal to the modal reflected power; therefore, these concepts can be used interchangeably.

Ideally, zero total reflected power for all possible excitation sets is needed to design an ideal TxArray coil that corresponds

F I G URE 9 Measured (left) and simulated (right) $\mathrm{B}_{1}^{+}$patterns of $\mathrm{CP}$ excitation mode at central axial plane (plane 2) for fabricated and simulated $2 \times$ 4-channel TxArray coil designed based on $\lambda$-opt (case 1) approach. Field patterns are normalized by square root of total incident power. In measured $\mathrm{B}_{1}^{+}$pattern, phase/ magnitude shimming was not performed

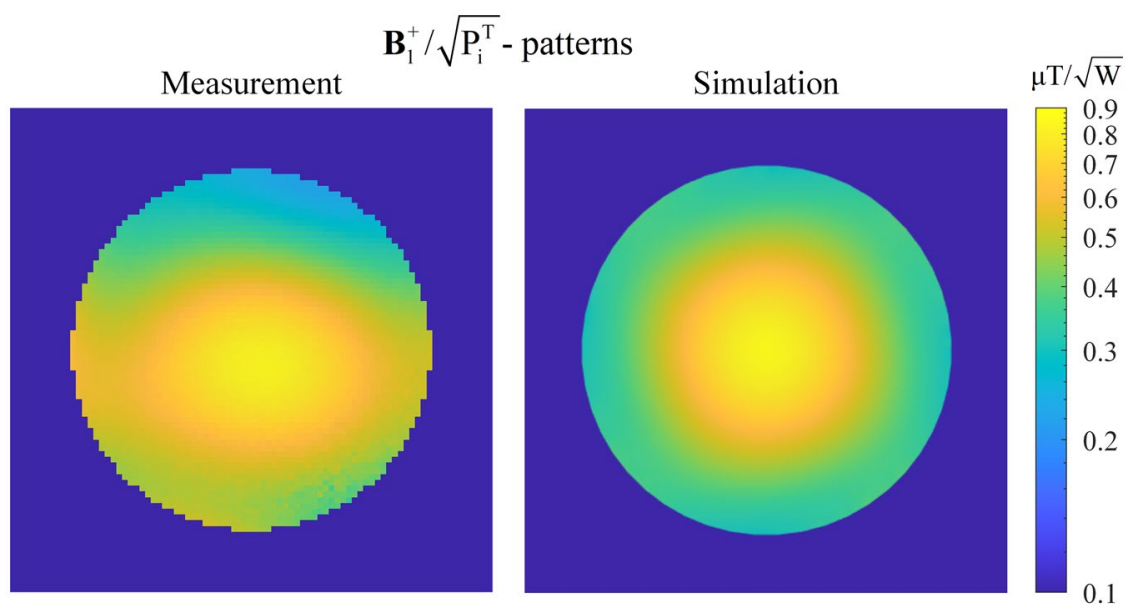


to a zero set of $\lambda_{n}$ values, which is possible only if the coil has a zero $\mathbf{S}$-matrix. This is also the ideal design target to achieve perfect matching and decoupling levels. Due to the practical imperfections, S-matrix cannot be zero; therefore, optimization algorithms can be configured and run to minimize the $\mathbf{S}$ matrix elements. In current practice, the magnitude of $\mathbf{S}$-matrix elements is minimized to match and decouple a TxArray coil. However, minimizing $\mathbf{S}$-matrix elements does not necessarily minimize the $\lambda_{n}$ values because, apart from the amplitude, the $\mathbf{S}$-matrix phase is also effective in determining the $\lambda_{n}$ values. The proposed eigenmode analysis looks at the $\mathbf{S}$-matrix as a whole, captures more than just looking at the $\mathbf{S}$-matrix elements individually. Hence, the minimization of the $\lambda_{n}$ values is a convenient approach for the design of TxArray coils.

The eigenmodes with high modal reflected power values hardly contribute to the transmission process and can, to a certain degree, be considered inefficient and impractical. The eigenmode analysis thus provided insight to determine the optimum number of transmit elements. Hence, the number of efficient eigenmodes might be considered as a critical metric to compare the performance of various TxArray coils. Therefore, this study focused mainly on designing TxArray coils by increasing the number of efficient eigenmodes. The set of eigenmodes that has low reflection is considered as the excitation space of the TxArray coil. In the design process, we want to expand the excitation space. Although there seems to be a limit in this, the coverage of this space can be modified. In our example designs, we made sure that $\mathrm{CP}$ excitation lies within this space. It should be noted that without limiting $\lambda_{C P}$, there are many design possibilities for the TxArray coils proposed in this study in which the CP mode does not lie within the low reflection excitation space. The future users of the proposed algorithm may add additional modes to the optimization process that are significant for the specific design.

For a predesigned TxArray coil, categorizing the efficient and inefficient eigenmodes limits the excitation space to the subset of eigenmodes. In our study, we used $50 \%$ as a reasonable number for this limit. In practice, this limit should be defined by the hardware limitations of the overall system, including the available power and the amount of tolerable reflected power. If users, for example, want to conduct RF shimming, the knowledge of the excitation space enables users to achieve a proper shim more rapidly within the power limits of the amplifiers.

Assessment of the total power absorbed by the phantom is critically essential. The total absorbed power by the object of interest and the conductor and radiation losses cannot be distinguished by merely assessing the scattering parameters. In the electromagnetic simulation, careful integration within the phantom is necessary. Furthermore, maximizing the power delivered to the object is not directly linked to generating the desired $\mathrm{B}_{1}^{+}$field. Linking the excitation space to these parameters is the subject of future research.

Although we conducted an eigenmode analysis of the S-matrix of TxArray coils, it was used earlier for other parameters. For example, the eigenmode analysis was applied to evaluate the SNR behavior of receive array coils, ${ }^{71,72}$ to improve parallel imaging performance, ${ }^{73}$ and to attain a homogeneous transmit excitation with low levels of specific absorption rate at ultrahigh field MRI. ${ }^{74}$ In this study, however, the eigenmode analysis is used to obtain power-efficient operation modes of TxArray coils.

\section{6 | CONCLUSION}

In this study, we examined the performance of a dual-row TxArray coil as an example to evaluate the effectiveness of the eigenmode analysis. We demonstrated that the structure chosen for the dual-row TxArray coil could act like a single-row degenerate birdcage TxArray coil by providing the $\mathrm{CP}$ excitation field patterns under certain circumstances (Supporting Information Figure S2). Consequently, this coil enables us to benefit from the advantages of the parallel transmit technology without losing the advantages of the conventional birdcage coil to obtain rather homogenous excitation patterns in a large volume.

For the analysis and design of the TxArray coils, the use of the modal reflected power concept is introduced. The capacitor values were found using our newly developed $\lambda$-opt approach. In this approach, the $\lambda_{n}$ values were minimized to increase the dimension of the excitation space (a subspace composed of the excitation eigenmodes with low total reflected power values). Additionally, the total reflected power for some critical modes of operations, such as the $\mathrm{CP}$ excitation mode, can be incorporated as a constraint in the optimization process. To examine the impact of the added constraint and weighting coefficients on the coil performance, 3 different minimization cases of the $\lambda$-opt approach were employed. To validate the effectiveness of the $\lambda$-opt approach, the optimal design performance of a dual-row TxArray coil achieved by the $\lambda$-opt method was compared with the optimal design obtained by the conventional minimization approach (S-opt). We showed that the proposed algorithm could increase excitation space, and some critical modes of operations can be achieved without significant reflection.

\section{DATA AVAILABILITY STATEMENT}

MatLab (MathWorks) codes that reproduce the results shown in Table 1 are available openly at https://github.com/ UMRAM-Bilkent/Eigenmode-Analysis. 


\section{ORCID}

\section{Ehsan Kazemivalipour (iD) https://orcid.} org/0000-0003-4221-2397

Alireza Sadeghi-Tarakameh (iD) https://orcid. org/0000-0001-5718-6553

Ergin Atalar ${ }^{(D)}$ https://orcid.org/0000-0002-6874-6103

\section{REFERENCES}

1. Adriany G, Van de Moortele P-F, Wiesinger F, et al. Transmit and receive transmission line arrays for 7 Tesla parallel imaging. Magn Reson Med. 2005;53:434-445.

2. Adriany G, Auerbach EJ, Snyder CJ, et al. A 32-channel lattice transmission line array for parallel transmit and receive MRI at 7 Tesla. Magn Reson Med. 2010;63:1478-1485.

3. Rietsch SHG, Orzada S, Bitz AK, et al. Parallel transmit capability of various RF transmit elements and arrays at 7T MRI. Magn Reson Med. 2018;79:1116-1126.

4. Avdievich NI, Hoffmann J, Shajan G, et al. Evaluation of transmit efficiency and SAR for a tight fit transceiver human head phased array at 9.4 T. NMR Biomed. 2017. https://doi.org/10.1002/ nbm. 3680

5. Sadeghi-Tarakameh A, DelaBarre L, Lagore RL, et al. In vivo human head MRI at 10.5T: A radiofrequency safety study and preliminary imaging results. Magn Reson Med. 2020;84:484-496.

6. Sadeghi-Tarakameh A, Adriany G, Metzger GJ, et al. Improving radiofrequency power and specific absorption rate management with bumped transmit elements in ultra-high field MRI. Magn Reson Med. 2020;84:3485-3493.

7. Sadeghi-Tarakameh A, Torrado-Carvajal A, Ariyurek C, et al. Optimizing the topography of transmit coils for SAR management. In Proceedings of the 26th Annual Meeting of ISMRM, Paris, France, 2018. p. 0297.

8. Sadeghi-Tarakameh A, Jungst S, Wu X, et al. A new coil element for highly-dense transmit arrays: An introduction to non-uniform dielectric substrate (NODES) antenna. In Proceedings of the 27th Annual Meeting of ISMRM, Montréal, Québec, Canada, 2019. p. 0732.

9. Van den Berg CAT, van den Bergen B, Van de Kamer JB, et al. Simultaneous B-1(+) homogenization and specific absorption rate hotspot suppression using a magnetic resonance phased array transmit coil. Magn Reson Med. 2007;57:577-586.

10. Katscher U, Bornert P. Parallel RF transmission in MRI. NMR Biomed. 2006; 19:393-400.

11. McElcheran CE, Golestanirad L, Iacono MI, et al. Numerical simulations of realistic lead trajectories and an experimental verification support the efficacy of parallel radiofrequency transmission to reduce heating of deep brain stimulation implants during MRI. Sci Rep. 2019. https://doi.org/10.1038/s41598-01838099-w

12. Childs AS, Malik SJ, O'Regan DP, et al. Impact of number of channels on RF shimming at 3T. MAGMA. 2013;26:401-410.

13. Kozlov M, Turner R. Analysis of RF transmit performance for a multi-row multi-channel MRI loop array at 300 and $400 \mathrm{MHz}$. Asia-Pacific Microwave Conference. 2011;2011:1190-1193.

14. Guérin B, Gebhardt M, Serano P, et al. Comparison of simulated parallel transmit body arrays at $3 \mathrm{~T}$ using excitation uniformity, global SAR, local SAR, and power efficiency metrics. Magn Reson Med. 2015;73:1137-1150.
15. Setsompop K, Wald LL, Alagappan V, et al. Parallel RF transmission with eight channels at 3 Tesla. Magn Reson Med. 2006;56:1163-1171.

16. Eryaman Y, Guerin B, Akgun C, et al. Parallel transmit pulse design for patients with deep brain stimulation implants. Magn Reson Med. 2015;73:1896-1903.

17. Guerin B, Angelone LM, Dougherty D, et al. Parallel transmission to reduce absorbed power around deep brain stimulation devices in MRI: Impact of number and arrangement of transmit channels. Magn Reson Med. 2020;83:299-311.

18. Kazemivalipour E, Keil B, Vali A, et al. Reconfigurable MRI technology for low-SAR imaging of deep brain stimulation at 3T: Application in bilateral leads, fully-implanted systems, and surgically modified lead trajectories. Neuroimage. 2019;199:18-29.

19. Golestanirad L, Kazemivalipour E, Keil B, et al. Reconfigurable MRI coil technology can substantially reduce RF heating of deep brain stimulation implants: First in-vitro study of RF heating reduction in bilateral DBS leads at 1.5 T. PLoS One. 2019;14:e0220043.

20. Roemer PB, Edelstein WA, Hayes CE, et al. The NMR phasedarray. Magn Reson Med. 1990;16:192-225.

21. Keil B, Alagappan V, Mareyam A, et al. Size-optimized 32-channel brain arrays for $3 \mathrm{~T}$ pediatric imaging. Magn Reson Med. 2011;66:1777-1787.

22. Sadeghi-Tarakameh A, Kazemivalipour E, Demir T, et al. Design of a degenerate birdcage radiofrequency transmit array coil for the magnetic resonance imaging using equivalent circuit model. In Proceedings of the 34th Annual Scientific Meeting of ESMRMB, Barcelona, Spain, 2017. p. 300-301.

23. Kazemivalipour E, Sadeghi-Tarakameh A, Yilmaz U, et al. A 12-channel degenerate birdcage body transmit array coil for $1.5 \mathrm{~T}$ MRI scanners. In Proceedings of the 26th Annual Meeting of ISMRM, Paris, France, 2018. p. 1708.

24. Avdievich NI, Pfrommer A, Giapitzakis IA, et al. Analytical modeling provides new insight into complex mutual coupling between surface loops at ultrahigh fields. NMR Biomed. 2017. https://doi. org/10.1002/nbm.3759

25. Kazemivalipour E, Sadeghi-Tarakameh E, Atalar E. Optimization of the degenerate birdcage transmit array coil for minimum coupling. In Proceedings of the 27th Annual Meeting of ISMRM, Montréal, Québec, Canada, 2019. p. 1604.

26. Kazemivalipour E, Sadeghi-Tarakameh A, Atalar E. Design of transmit array coils for MRI by minimizing the modal reflection coefficients. In Proceedings of the ISMRM \& SMRT Virtual Conference \& Exhibition, 2020. p. 0762.

27. Jevtic J. Ladder networks for capacitive decoupling in phasedarray coils. In Proceedings of the 9th Annual Meeting of ISMRM, Glasgow, Scotland. 2001. p. 17.

28. Lee RF, Giaquinto RO, Hardy CJ. Coupling and decoupling theory and its application to the MRI phased array. Magn Reson Med. 2002;48:203-213.

29. Mahmood Z, Guérin B, Adalsteinsson E, et al. An automated framework to decouple pTx arrays with many channels. In Proceedings of the 21st Annual Meeting of ISMRM, Salt Lake City, UT, 2013. p. 2722.

30. Avdievich NI, Giapitzakis IA, Pfrommer A, et al. Decoupling of a double-row 16-element tight-fit transceiver phased array for human whole-brain imaging at 9.4 T. NMR Biomed. 2018;31:e3964.

31. Hoult DI. The principle of reciprocity in signal strength calculations: A mathematical guide. Concepts Magn Reson. 2000;12:173-187. 
32. Connell IRO, Gilbert KM, Abou-Khousa MA, et al. MRI RF array decoupling method with magnetic wall distributed filters. IEEE Trans Med Imaging. 2015;34:825-835.

33. Kozlov M, Turner R. Analysis of RF transmit performance for a 7T dual row multichannel MRI loop array. In Proceedings of the Annual International Conference of the IEEE Engineering in Medicine and Biology Society (EMBC), 2011. p. 547-553.

34. Guérin B, Setsompop K, Ye H, et al. Design of parallel transmission pulses for simultaneous multislice with explicit control for peak power and local specific absorption rate. Magn Reson Med. 2015;73:1946-1953.

35. Guérin B, Gebhardt M, Cauley S, et al. Local specific absorption rate (SAR), global SAR, transmitter power, and excitation accuracy trade-offs in low flip-angle parallel transmit pulse design. Magn Reson Med. 2014;71:1446-1457.

36. Brunner DO, Pruessmann KP. Optimal design of multiple-channel RF pulses under strict power and SAR constraints. Magn Reson Med. 2010;63:1280-1291.

37. Vaughan JT, Garwood M, Collins CM, et al. 7T vs. 4T: RF power, homogeneity, and signal-to-noise comparison in head images. Magn Reson Med. 2001;46:24-30.

38. van den Bergen B, van den Berg CAT, Klomp DWJ, et al. SAR and power implications of different RF shimming strategies in the pelvis for 7T MRI. J Magn Reson Imaging. 2009;30:194-202.

39. Kozlov M, Turner R. Effects of tuning condition, head size and position on the SAR of a MRI dual-row transmit array at $400 \mathrm{MHz}$. In Proceedings Progress in Electromagnetics Research Symposium (PIERS), Taipei, Taiwan. 2013. p. 422-426.

40. Yan X, Ole Pedersen J, Wei L, et al. Multichannel double-row transmission line array for human MR imaging at ultrahigh fields. IEEE Trans Biomed Eng. 2015;62:1652-1659.

41. Shajan G, Kozlov M, Hoffmann J, et al. A 16-channel dual-row transmit array in combination with a 31-element receive array for human brain imaging at 9.4 T. Magn Reson Med. 2014;71: 870-879.

42. Hoffmann J, Shajan G, Scheffler K, et al. Numerical and experimental evaluation of RF shimming in the human brain at $9.4 \mathrm{~T}$ using a dual-row transmit array. MAGMA. 2014;27: 373-386.

43. Alagappan V, Nistler J, Adalsteinsson E, et al. Degenerate mode band-pass birdcage coil for accelerated parallel excitation. Magn Reson Med. 2007;57:1148-1158.

44. Stara R, Tiberi G, Morsani F, et al. A degenerate birdcage with integrated Tx/Rx switches and butler matrix for the human limbs at 7 T. Appl Magn Reson. 2017;48:307-326.

45. Ibrahim TS, Lee R, Baertlein BA, et al. Computational analysis of the high pass birdcage resonator: Finite difference time domain simulations for high-field MRI. Magn Reson Imaging. 2000;18:835-843.

46. Golestanirad L, Kazemivalipour E, Lampman D, et al. RF heating of deep brain stimulation implants in open-bore vertical MRI systems: A simulation study with realistic device configurations. Magn Reson Med. 2020;83:2284-2292.

47. Kazemivalipour E, Vu J, Lin S, et al. RF heating of deep brain stimulation implants during MRI in $1.2 \mathrm{~T}$ vertical scanners versus 1.5 T horizontal systems: A simulation study with realistic lead configurations. In Proceedings of the 42nd Annual International Conference of the IEEE Engineering in Medicine \& Biology Society (EMBC), Montréal, Québec, Canada, 2020. p. 6143-6146.

48. Pozar DM. Microwave Engineering. Hoboken, NJ: Wiley; 2012.
49. Volmer C, Weber J, Stephan R, et al. An eigen-analysis of compact antenna arrays and its application to port decoupling. IEEE Trans Antennas Propag. 2008;56:360-370.

50. Irteza S, Schäfer E, Stephan R, et al. Compact antenna array receiver for robust satellite navigation systems. Int J Microw Wirel T. 2015;7:735-745.

51. Horn RA, Johnson CR. Matrix Analysis. Cambridge, UK; New York, NY: Cambridge University Press; 1985.

52. Lay DC, Lay SR, McDonald J. Linear Algebra and its Applications. Boston, MA: Pearson; 2020.

53. Trefethen LN, Bau D. Numerical Linear Algebra. Philadelphia, PA: Society for Industrial and Applied Mathematics; 1997.

54. Kozlov M, Turner R. Comprehensive analysis of transmit performance for an 8-element loop MRI RF coil at $300 \mathrm{MHz}$. In Proceedings of the 40th European Microwave Conference, 2010. p. 328-331.

55. Kozlov M, Lucano E, Angelone LM. Effects of tuning conditions on near field of MRI transmit birdcage coil at $64 \mathrm{MHz}$. In Proceedings of the 38th Annual International Conference of the IEEE Engineering in Medicine and Biology Society (EMBC), 2016. pp. 6242-6245.

56. Sadeghi-Tarakameh A, Kazemivalipour E, Demir $\mathrm{T}$, et al. Accelerating the cosimulation method for fast design of transmit array coils: An example study on a degenerate birdcage coil. In Proceedings of the 26th Annual Meeting of ISMRM, Paris, France, 2018. p. 4404.

57. Nistler J, Kurth R, Vester M, et al. B1 inhomogenisation using a multichannel transmit array. In Proceedings of the 14th Annual Meeting of ISMRM, Seattle, WA, USA. 2006.

58. Sadeghi-Tarakameh A, Kazemivalipour E, Gundogdu U, et al. Accelerating the co-simulation method for the design of transmit array coils for MRI. MAGMA. 2020. https://doi.org/10.1007/s1033 4-020-00858-0

59. Kazemivalipour E, Sadeghi-Tarakameh A, Yilmaz U, et al. Design of an 8-channel transmit array coil using the equivalent circuit model of the manufactured structure. In Proceedings of the 28th Annual Meeting of ISMRM, Virtual, 2020. p. 0758.

60. Balanis CA. Advanced Engineering Electromagnetics. Hoboken, NJ: John Wiley \& Sons; 2012.

61. Kozlov M, Turner R. Fast MRI coil analysis based on 3-D electromagnetic and RF circuit co-simulation. J Magn Reson. 2009;200:147-152.

62. Hayes CE, Eash MG, inventors; General Electric Co, assignees. Shield for decoupling RF and gradient coils in an NMR apparatus. US patent 4642569A. February 10, 1987.

63. Gurler N, Ider YZ. Gradient-based electrical conductivity imaging using MR phase. Magn Reson Med. 2017;77:137-150.

64. Gabriel C, Gabriel S, H. Grant E, et al. Dielectric parameters relevant to microwave dielectric heating. Chem Soc Rev. 1998;27:213-223.

65. Winkler SA, Rutt BK. Practical methods for improving B-1(+) homogeneity in 3 Tesla breast imaging. J Magn Reson Imaging. 2015;41:992-999.

66. Sacolick LI, Wiesinger F, Hancu I, et al. B1 mapping by BlochSiegert shift. Magn Reson Med. 2010;63:1315-1322.

67. Turk EA, Ider YZ, Ergun AS, et al. Approximate Fourier domain expression for Bloch-Siegert shift. Magn Reson Med. 2015;73:117-125.

68. Ludwig AC. Mutual coupling, gain and directivity of an array of 2 identical antennas. IEEE Trans Antennas Propag. 1976;24:837-841. 
69. Chae SH, Oh SK, Park SO. Analysis of mutual coupling, correlations, and TARC in WiBro MIMO array antenna. IEEE Antennas Wirel Propag Lett. 2007;6:122-125.

70. Hannula J-M, Saarinen T, Holopainen J, et al. Frequency reconfigurable multiband handset antenna based on a multichannel transceiver. IEEE Trans Antennas Propag. 2017;65: 4452-4460.

71. King SB, Varosi SM, Duensing GR. Eigenmode analysis for understanding phased array coils and their limits. Concepts Magn Reson Part B Magn Reson Eng. 2006;29b:42-49.

72. King SB, Varosi SM, Duensing GR. Optimum SNR data compression in hardware using an eigencoil array. Magn Reson Med. 2010;63:1346-1356.

73. Wang CS, Qu P, Shen GX. Potential advantage of higher-order modes of birdcage coil for parallel imaging. J Magn Reson. 2006;182:160-167.

74. Santini T, Zhao Y, Wood S, et al. In-vivo and numerical analysis of the eigenmodes produced by a multi-level Tic-Tac-Toe head transmit array for 7 Tesla MRI. PLoS One. 2018;13:e206127.

\section{SUPPORTING INFORMATION}

Additional Supporting Information may be found online in the Supporting Information section.

FIGURE S1 A, EM simulation models and B, general schematic of four high-pass birdcage coils. All coils have the same dimensions, are enclosed with the same cylindrical
RF shields, and are loaded by the same uniform cylindrical phantoms

FIGURE S2 Surface current density of the $2 \times 4$-channel TxArray coil designed based on the $\lambda$-opt (case\#2) approach and the $\mathrm{B}_{1}^{+}$-patterns within the phantom for the $\mathrm{CP}$ excitation mode at three different axial planes. Surface current densities and $\mathrm{B}_{1}^{+}$-patterns are normalized by the square root of the total incident power

TABLE S1 Optimized capacitor values for the simulated TxArray coils and high-pass birdcage coils

TABLE S2 Optimized capacitor values for the fabricated and simulated $2 \times 4$-channel TxArray coils

TABLE S3 Sensitivity of the eigenvalues to the conductivity of the phantom

TABLE S4 Sensitivity of the eigenvalues to the phantom diameter

How to cite this article: Kazemivalipour E, SadeghiTarakameh A, Atalar E. Eigenmode analysis of the scattering matrix for the design of MRI transmit array coils. Magn Reson Med. 2021;85:1727-1741. https:// doi.org/10.1002/mrm.28533 